\title{
Anisotropic optical response of GaN and AlN nanowires
}

\section{A. Molina-Sánchez}

Instituto de Ciencia de Materiales, Universidad de Valencia, E-46071 Valencia, Spain Physics and Material Sciences Research Unit, Université du Luxembourg, Luxembourg

\section{A. García-Cristóbal}

Instituto de Ciencia de Materiales, Universidad de Valencia, E-46071 Valencia, Spain

\begin{abstract}
We present a theoretical study of the electronic structure and optical properties of free-standing GaN and AlN nanowires. We have implemented the empirical tight-binding method, with an orbital basis $s p^{3}$, that includes the spin-orbit interaction. The passivation of the dangling bonds at the free surfaces is also studied together with the effects on the nanowire electronic structure. For both GaN and AlN nanowires, we have found a remarkable anisotropy of the optical absorption when the light polarization changes, showing in the case of GaN a dependence on the nanowire size.
\end{abstract}




\section{Introduction}

Recent advances in the growth techniques of nitrides III-V semiconductor nanowires (NW) have focused the attention in these one-dimensional nanostructures due to their potential applications in optoelectronic.[1] The plasma-assisted molecular beam epitaxy is capable of fabricating free-standing nanowires of $\operatorname{GaN}[2,3]$ and $\operatorname{InN},[4]$ and with the physical vapor transport have fabricated AlN nanowires, $[5,6,7]$ all of them with high crystalline quality. Several differences with the still widely investigated two-dimensional layers and quantum dots should be remarked. Firstly, nanowires grow strain-free (except maybe at their base), and with a minimal presence of dislocations or defects along the main structure. Moreover, contrary to quantum dots, that are usually immersed in a matrix of another material, free-standing NWs have the advantage that they can be easily separated from the substrate and dispersed in a surface. Thus, transport measurements in single NWs can be performed, [8, 9] as well as polarization-resolved spectroscopy. [10] In particular, III-V semiconductor nanowires are suitable for band gap engineering. Thus, InN has a band gap of $0.67 \mathrm{eV}(1852 \mathrm{~nm})$, whereas GaN and AlN have a band gap of $3.5 \mathrm{eV}(355 \mathrm{~nm})$ and $6.2 \mathrm{eV}(200 \mathrm{~nm})$, respectively. This opens the possibility of covering the whole solar spectrum by an appropriate alloying, [12] being a focus of research for the development of photovoltaic cells,[13] among others applications as white-emitting devices[11] or field-effect transistors.[14] Besides, the refinement of the growth techniques leads the reduction of the NW size until quantum confinement effects emerge.[15, 17, 16] In this context, theory and numerical simulations play a crucial role in the explanation of NWs properties and a better understanding of the observed phenomena. Theoretical studies are also required to contribute in the interpretation of the experiments or to investigate the trends in the optical properties, helping in the design of a more efficient fabrication of devices.

In the theoretical study of nanostructures we can find two main approaches to board the problem, by means of atomistic methods or with continuous methods (envelope function approximation).[18, 20, 21, 19] This last methodology could omit the truth symmetry of the system, missing important effects.[22, 23] This problem related to symmetry is automatically resolved in the atomistic approaches. Thus, the most accurate atomistic methodology are those funded in ab initio, and already implemented for nanowires of $\mathrm{GaN}, \mathrm{ZnO},[24] \mathrm{AlN}[25]$ and $\mathrm{ZnO} / \mathrm{ZnS}$.[26] However, such methods are very time demanding when dealing with system composed by hundred of atoms, that preclude of a systematic study of nanowire properties as a function of size or/and morphology. Alternatively, atomistic empirical methods capture also automatically the symmetry and can deal with system of thousands of atoms in a more effective way than $a b$ initio methods. Moreover, such methods are desirable to bridge the gap between the first principles methods used for very thin nanowires and the effective function approximation approaches, suitable for large nanostructures. Specifically, the empirical tight-binding method has shown to describe accurately nanostructures within reasonable computational costs. [27, 28, 29, 30] 
In this work, we present a comparative study of the electronic and optical properties of GaN and AlN nanowires, implementing the tight-binding model as described in Refs. [31] and [32]. The treatment of the lateral free surfaces and the consequent problem of passivation has also been studied in detail, explaining how surface states influence on the electronic structure. Moreover, we have also investigated the dependence of the nanowires properties for varying sizes, with special attention to the changes in the absorption polarization. This paper is organized as follows: the Section II (Theory) is devoted to explain the main features of the model, using an example of the bulk band structure and giving details of the surface modeling, Section III (Numerical Results) shows the calculation for GaN and AlN, and in Section IV (Conclusions) main results have been discussed.

\section{The tight-binding method}

\subsection{Formulation}

The tight-binding method is a well-known tool applied to the study of the electronic and optical properties, among others, of semiconductors nanostructures. A great variety of implementations of this method are available in the literature, depending on the basis functions size or the number of neighbor atoms for which the interaction is taken into account. Thus, we can find implementations with a small basis $\left(s_{c} p_{a}^{3}\right)$ in the modeling of GaN/AlN quantum dots, with a good agreement at $\Gamma$-point, in comparison with results of the $\boldsymbol{k} \cdot \boldsymbol{p}$ method, [34] or studies of nitride bulk band structure with a very large basis, $s p s^{*} d$, of twenty orbitals per atom, giving a precise description of most of the points of the Brillouin zone.[35, 36]

In this work, we employ an $s p^{3}$ implementation that uses eight orbitals per atom (the spin-orbit interaction is included following Ref. [41]), and limits the interaction to nearest neighbors. This basis gives a good description of the band structure near the $\Gamma$-point point, and reproduces reasonably well the other bands. Within this method, the wave function of a single electron is generally written as:

$$
\Psi_{k}^{n}(\boldsymbol{r})=\sum_{\alpha, j, \sigma} A_{\alpha, j, \sigma}^{n}(k) \phi_{\alpha, j, \sigma}\left(\boldsymbol{r}-\boldsymbol{r}_{\alpha}\right)
$$

where $\phi_{\alpha, j, \sigma}\left(\boldsymbol{r}-\boldsymbol{r}_{\alpha}\right)$ is the atomic orbital of symmetry $j$ and $\operatorname{spin} \sigma\left(j=s, p_{x}, p_{y}, p_{z}\right.$, $\sigma= \pm 1 / 2$ ), corresponding to atom $\alpha$ centered at $\boldsymbol{r}_{\alpha}$. Upon introducing the expansion of Eq. (1) into the Schrödinger equation and following the standard procedure explained elsewhere, $[37,38]$ a Hamiltonian eigenvalue problem is obtained. The matrix diagonalization gives the electronic energies $\epsilon^{n}(k)$ and the wave functions expressed in terms of the coefficients $A_{\alpha, j, \sigma}^{n}(k)$. The Hamiltonian matrix elements can be expressed in term of intra-atomic parameters (diagonal) $E_{\alpha, j}$, describing the atomic energy of each orbital corrected by the crystal environment, and inter-atomic parameters (off-diagonal) $V_{j, j^{\prime}}^{\alpha, \alpha^{\prime}}$ responsible for the interaction between neighbor atoms. Taking advantage of 
the orbital symmetries, one can write the Hamiltonian matrix with a reduced set of parameters and the director cosines between atoms.[31] Mention that the parameter sets are not univocal, and they are in constant revision. In this work, the parameters are taken from Ref. [43] for GaN and Ref. [32] for AlN. At this point, it is worth to mention that the $s p^{3}$ basis in the nearest neighbor approximation cannot reproduce the crystal-field splitting, and gives in the absence of spin-orbit interaction a six-fold degeneracy at the top of the valence band.[32] The reason is the equivalence between wurtzite and zinc-blende at the nearest neighbors approximation, assuming a equidistant tetragonal bond. The introduction of second and farther neighbors would palliate this deficiency.[40] An alternative to the addition of farther neighbors, is to distinguish by hand between the $p_{x}-p_{y}$ and $p_{z}$ orbitals. This ad hoc modification is performed on the on-site energy $E_{a, p_{z}} \neq E_{a, p_{x}}=E_{a, p_{y}}$, taking the values of $E_{a, p_{z}}^{a}(\mathrm{GaN})=1.2352$ $\mathrm{eV}$, and $E_{a, p_{z}}^{a}(\mathrm{AlN})=3.6212 \mathrm{eV}$. With this approach, and taking account the spinorbit interaction, we can reproduce the known three edge structure of the valence band characteristic of wurtzite semiconductors.[42] Thus, we achieve an optimal compromise between an accurate description and a low computational cost.

Another important aspect of the electronic states is their symmetry character, that we quantify here by the projection of the wave function (of state $n$ ) over the atomic orbital $j, \sigma$

$$
\phi_{j}^{n}=\sum_{\alpha, \sigma}\left|\left\langle\phi_{\alpha, j, \sigma} \mid \Psi_{k}^{n}\right\rangle\right|^{2}=\sum_{\alpha, \sigma}\left|A_{\alpha, j, \sigma}^{n}\right|^{2} .
$$

The set of numbers $\phi_{j}^{n}$ for a given state summarizes the relevant information about the symmetry of the state and satisfies $\sum_{j} \phi_{j}^{n}=1$. Given the in-plane isotropy is convenient to introduce the symbol $\phi_{p_{\perp}}^{n}=\phi_{p_{x}}^{n}+\phi_{p_{y}}^{n}$. Usually one of these numbers is dominant (say $>0.5$ ), and in this situation we say that the state has predominant $j$-character.

In order to illustrate the tight-binding formulation and the notation defined above in a practical example, the valence bands corresponding to GaN and AlN are presented in Fig. 1. The valence band edge energies are labeled, in increasing energy order, as $E_{1}, E_{2}, E_{3}$. The dominant character of the corresponding wave functions is indicated in parenthesis. Note that the GaN and AlN valence bands differ notably in spite of sharing the same crystalline structure. In the case of $\mathrm{GaN}$, the two top most valence bands, $E_{1}, E_{2}$ have a predominant $p_{\perp}$-character near the zone center, and are separated mainly because of the spin-orbit coupling. The crystal-field interaction splits the band $E_{3}$ (with main contribution $p_{z}$ ) to lower energies. We find all these three states within the range of $30 \mathrm{meV}$. [45] In the case of AlN, the top valence band $E_{1}$ has now major composition $p_{z}$, and the next bands, $E_{2}$ and $E_{3}$, composed by $p_{x}$ and $p_{y}$ orbitals, are placed $100 \mathrm{meV}$ below. This property has been confirmed by experiments of angledependent photoluminescence performed in a C-oriented AlN surface.[46] However, the exact values of spin-orbit and crystal-field splitting are still controversial due to the difficulty in obtaining accurate measurements. $[45,47,48]$ The reference to the bulk 

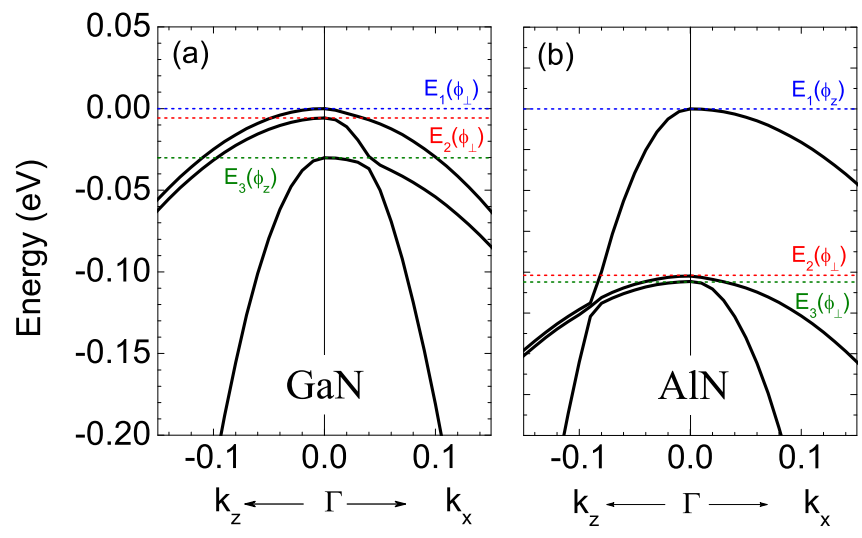

Figure 1. Valence Band of GaN (a) and AlN (b) calculated with the tight-binding described in the text. The band edge energies are labeled in increasing order of energy, as $E_{1}, E_{2}$ and $E_{3}$ and the dominant character (here $\phi_{j}>0.99$ ) is also given in parenthesis.

band structure and the notation defined here will facilitate the interpretation of the nanowire band structure and the optical response to be described below.

\subsection{Nanowires modeling, surfaces states and passivation}

We now change to the description employed in the modeling of nanowires. NWs are oriented along the $\mathrm{C}$-axis and infinite in length. We assume a hexagonal cross section, with (1100) (M-plane) facets. This is the most usual shape adopted by GaN NWs grown by plasma-assisted molecular beam epitaxy technique. [50, 49] This NW faceting has also been supported by theoretical calculations, [52] showing a lower formation energy for (1100) than $(11 \overline{2} 0)$ surfaces. A sketch of the modeled NW together with the supercell used for the calculation are shown in Fig. 2. Note that with the atomistic tight-binding model we can reproduce the exact shape, and consequently, the correct symmetry of the nanowire.

Another important issue concerns the NW free surface. First principles studies show a superficial rearrangement of the atoms close to the surface, with the corresponding modification of interatomic distances of these atoms. [25] It is out of the scope of this paper to discuss in detail how this perturbation modifies the electronic structure. Moreover, for large enough NWs, the volume-to-surface ratio is high enough so that inner atoms are unaffected by the surface and dominate the electronic and optical properties. Therefore, all the atoms in the modeled NWs are supposed to arrange according the bulk wurtzite structure. Nevertheless, the presence of free surfaces (see Fig. 2) implies the existence of dangling bonds for the surface atoms. These bonds are passivated within the theoretical model with pseudo-hydrogen atoms.[53] However, the application of the passivation has to be carried out carefully. Spurious surface states can appear within the band gap, making difficult both the identification of NW states and the computational task. The passivating pseudo-hydrogen atoms are supposed 


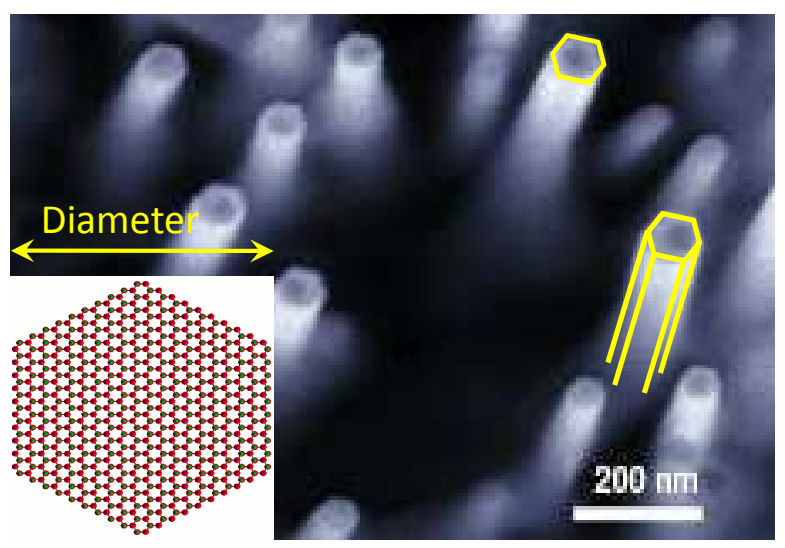

Figure 2. Scanning electron microscopy image of GaN nanowires grown by metalorganic chemical vapour deposition (Ref. [51]) and a sketch of the modeled nanowire. A ball-and-stick representation of the NW supercell is also displayed as an inset (dangling bonds are not drawn).

to interact only through their s-orbital with the NW atoms, and the corresponding interatomic parameters are obtained with the Harrison rule's: $[54,27]$

$$
V_{H}=\left(\frac{d_{0}}{d_{H}}\right)^{n_{d}} V_{0}
$$

where $d_{H}$ is the distance of the pseudo-hydrogen atom to the wurtzite atom, $d_{0}$ is the inter-atomic distance of wurtzite atoms, the exponent $n_{d}$ is characteristic of the material, and $V_{0}$ is the interatomic matrix elements. Here it is critical the choice of $d_{H}$ and $n_{d}$ to avoid the appearance of spurious states. For the sake of simplicity we assume $d_{H}=\frac{1}{2} d_{0}$ for all the dangling bonds, independently of the atomic species. The exponent $n_{d}$ enters here as a fitting parameter. The interaction between different hydrogen atoms is disregarded. The influence of the parameter $n_{d}$ on the NW band structure is illustrated in Fig. 3 through an example for GaN NWs. Figure 3 shows the energy of the first conduction band state, $c_{1}$, as a function of the NW size, for several values of the exponent $n_{d}$. It is important to note that for all the $n_{d}$ shown here, there are no surface states within the band gap at all. However, smaller values of $n_{d}$, or distances $d_{H}$ too large, could lead to the presence of states within the band gap. In Fig. 3, we see that the sensitivity of the $c_{1}$ energy to the parameter $n_{d}$ is reduced as the NW size increases, the change in the energy being negligible for diameters $>6 \mathrm{~nm}$. This is consistent with the fact that for larger NW cross sections, the surface effects are less important. Moreover, there is no hybridization between the conduction states and the surface states. In the inset of Fig. 3 we have fixed the NW diameter to 1.5 $\mathrm{nm}$, and plotted the energy of the state $c_{1}$ versus $n_{d}$. For $n_{d}>6$, a saturation of the energy is observed, reminiscent of the hard-wall condition (infinite barrier), often used in the effective-mass approximation. Concerning the valence band states, they do not show significant changes in their energy in the range of the parameter $n_{d}$ studied here. 


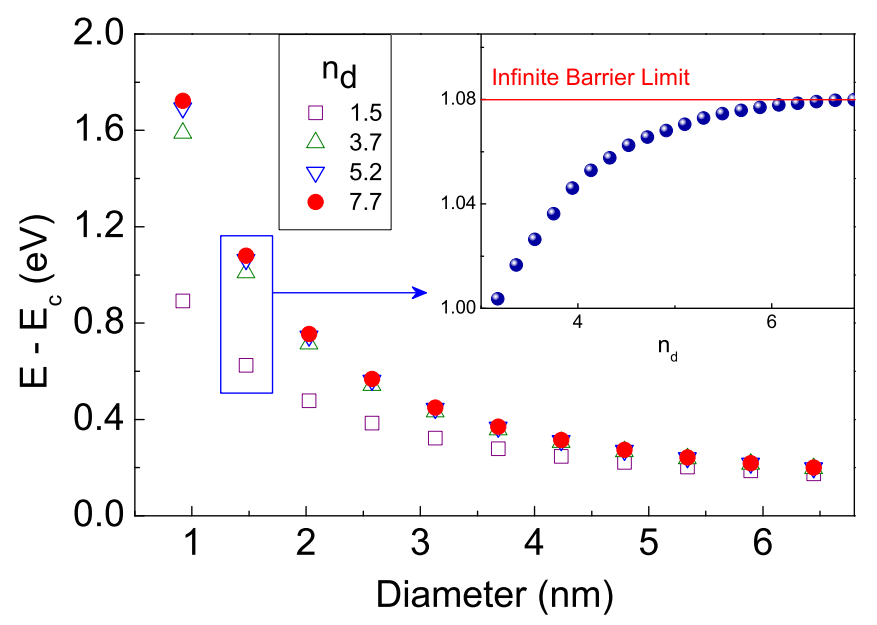

Figure 3. Size dependent of the energy of the first conduction band state for a GaN NW for different values of $n_{d}$. The distance $d_{H}$ is set to $\frac{1}{2} d_{0}$. In the inset, the dependence of the energy versus $n_{d}$ is represented for a diameter $1.5 \mathrm{~nm}$. $E_{c}$ is the GaN bulk conduction band edge energy.

This is again understandable since the interaction of the hydrogen atoms with $p$ orbitals takes place only through the parameters $V_{s_{c} p_{a}}$ and $V_{s_{a} p_{c}}$. In calculations where the passivation is not implemented and a bare NW is calculated, states spatially localized at the NWs surface and with energy at the bandgap appear. A realistic description of such states needs of a more sophisticated approach and eventually an optimization of the interatomic distances, which is out of the scope of this work. Moreover, in terms of computational efficiency, such surfaces states makes difficult the calculation and the identification of the confined NW wave functions, due to the addition of states in the same energy range.

The optical absorption spectrum $\alpha(\hbar \omega)$, for a given light-polarization $\boldsymbol{e}$, can be now computed directly by means of the expression:

$$
\alpha(\hbar \omega) \propto \sum_{n_{c}, n_{v}} \int_{B Z} f_{n_{c}, n_{v}}^{e}(k) \delta\left(\hbar \omega-\left(\epsilon_{n_{c}}(k)-\epsilon_{n_{v}}(k)\right)\right) d k
$$

where:

$$
f_{n_{c}, n_{v}}^{e}(k)=\frac{\left|\left\langle\Psi_{n_{c}}|\boldsymbol{e} \cdot \boldsymbol{p}| \Psi_{n_{v}}\right\rangle\right|^{2}}{\epsilon_{n_{c}}-\epsilon_{n_{v}}},
$$

is the oscillator strength for a transition from the valence band state $\left(n_{v}, k\right)$ to the conduction band $\left(n_{c}, k\right)$ and calculated following Ref. [44] which does not introduce additional parameters in the calculation. The delta function is replaced by a Lorentz's function of width $10 \mathrm{meV}$ in all the calculations, and the integral is solved by a proper discretization. It is also worth to mention that the Hamiltonian matrix constructed from the given supercell leads to a very large and sparse matrix. Since we are interested in few conduction and valence band states the Hamiltonian is shifted by an energy $E_{0}$ 
conveniently chosen within GaN bandgap:

$$
\left(H-E_{0}\right) \Psi_{k}^{n}=\left(E-E_{0}\right) \Psi_{k}^{n}
$$

This eigenvalue problem is solved by using the ARPACK subroutines, based in the Arnoldi algorithm.[55]

\section{Numerical Results}

In this section we present numerical results for the electronic structure and optical absorption spectrum of GaN and AlN NWs. In order to visualize the results, it is convenient to introduce the charge density (note that it loses the character state information), that is the sum of the contributions of the orbitals on their corresponding atomic site:

$$
\rho^{n}\left(\boldsymbol{r}_{\boldsymbol{\alpha}}\right)=\left\langle\Psi_{k}^{n} \mid \Psi_{k}^{n}\right\rangle=\sum_{j}\left|A_{\alpha, j}^{n}\right|^{2}
$$

\subsection{GaN nanowires}

We start by analyzing the influence of the NW diameter on the conduction band energy spectrum. In Fig. 4 we present the variation with the nanowire diameter of the energy of the first five $\mathrm{CB}$ states at $k_{z}=0$ (symbols). In the absence of spin-orbit interaction there would be an exact degeneracy between orbitally symmetric (equivalent) states. This degeneracy is broken in our calculations including the spin-orbit interaction, and the states labeled $\left(c_{2}, c_{3}\right)$ and $\left(c_{4}, c_{5}\right)$ are no longer degenerate. Nevertheless, the corresponding splitting $\epsilon_{3}^{(c)}-\epsilon_{2}^{(c)}$ and $\epsilon_{5}^{(c)}-\epsilon_{4}^{(c)}$ are so small $(<5 \mathrm{meV})$ that cannot be appreciated in Fig. 4. It must be noted that there a double Kramers degeneracy affecting every NW energy eigenstate. The wide bandgap of GaN prevents a strong coupling between the conduction and valence bands, being the dispersion of the conduction band around the $\Gamma$-point quasi parabolic. It is also noticeable that the confinement energy of the lowest $\mathrm{CB}$ state, $\epsilon_{1}^{(c)}-E_{c}$, remains relatively large $(>20 \mathrm{meV})$ up to the largest diameters of $20 \mathrm{~nm}$ considered in this work. The CB energies exhibit a monotonous decreasing evolution with the NW diameter, without any crossing or anti-crossing effects. This is related to the rather simple nature of the bulk conduction band which near the zone-center has a predominant $s$-character $\left(\phi_{s}>99 \%\right)$ and presents a nearly perfect parabolic behavior across most of the Gamma-A line of the Brillouin zone. However, this dependence of the energies does not coincide with the dependence proportional to $d^{-2}$ predicted by the simple effective-mass approximation for a cylindrical NW of diameter $d$. We have fitted the first state in Fig. 4 (triangles) to the function:

$$
\epsilon_{c_{1}}=E_{c}+\frac{a}{d^{b}} \quad(\text { in } \mathrm{eV})
$$


and obtained the parameters: $E_{c}=3.5, a=1.80 \pm 0.04$ and $b=1.48 \pm 0.02$. The $s$ orbital contribution for each state is also presented by interpolated lines in Fig. 4. As in bulk, the NW states have predominant $s$-character, and only for very narrow wires the $s$-orbital contribution of the ground CB state falls below $95 \%$. In comparison with the $a b$ initio calculations of Ref. [24], our results for the same size shows a larger value (5.4 $\mathrm{eV}$ tight-binding and $4.8 \mathrm{eV}$ ab initio), which is probably due to the underestimation of the bandgap value given by the local density approximation. Thus, tight-binding calculations should be compared with GW calculations or with confinement energy, as done in Ref. [30].

We now turn to the analysis of the VB electronic structure. As opposed to what happens in the $\mathrm{CB}$ electronic structure, the symmetry mixing and spin-orbit coupling within the bulk VB (see Fig. 1) together with the weaker confinement effects (due to larger effective masses) will cause a complicated size dispersion of the VB energy level structure of the GaN NW, as will be shown below. In particular, it becomes impossible to identify quasi-degenerate pairs of states as those shown in Fig. 4. Therefore, we have represented in Fig. 5 the energy levels of a set of representative $k_{z}=0 \mathrm{VB}$ states. More precisely, we show the 20 highest-energy levels (labeled $v_{1}$ to $v_{20}$ ) for every nanowire diameter, as obtained from the numerical diagonalization, irrespective of their symmetry properties. Thus, Fig. 5 gives an overall view of the confinement effects. For the largest NW analyzed (diameter $=17.5 \mathrm{~nm}$ ), the set of 20 levels spans an energy range of around $28 \mathrm{meV}$, with the highest one showing a confinement energy of around $8 \mathrm{meV}$. On the other hand, for a small radius NW, say $5.3 \mathrm{~nm}$ in diameter, the levels span a range of 130 $\mathrm{meV}$, the highest one being $48 \mathrm{meV}$ below the bulk VB band edge. We have also studied the symmetry of the wave functions. For the largest NW, states with energy above the bulk crystal-field splitting energy (blue dashed line) exhibit a definite character $p_{x, y}$, that allows to make an approximate correspondence, identification as confined states derived from the GaN bulk band edges $E_{1}$ and $E_{2}$. Nevertheless, as the NW size is decreased, the complex interplay between VB mixing and confinement mentioned above precludes a systematic interpolation of the energy levels that could allow to represent the energy spectrum in Fig. 5 as a series of continuous size dispersion curves.

This would only be achieved after an exhaustive group theoretical analysis of the atomistic eigenvalue problem, that would allow to assign the exact symmetry (group representation) of every state. Not having done this analysis, which is outside the scope of this work, we must content ourselves with a state-by-state individual analysis of the eigenstates obtained numerically. Following this procedure, after a careful examination of the wave functions of the highest-energy VB states we have been able to trace various interpolating lines connecting states with the same global symmetry. This simple exercise already shows the existence of both crossing (between different symmetry states) and anti-crossing effects (between same symmetry effects), which result in a nontrivial, non monotonous evolution of the size dispersion curves. In particular, a rather noticeable behavior arises as a consequence of this level interaction: The two highest energy size dispersion curves cross at around $5 \mathrm{~nm}$, so that the highest energy VB state (ground hole 


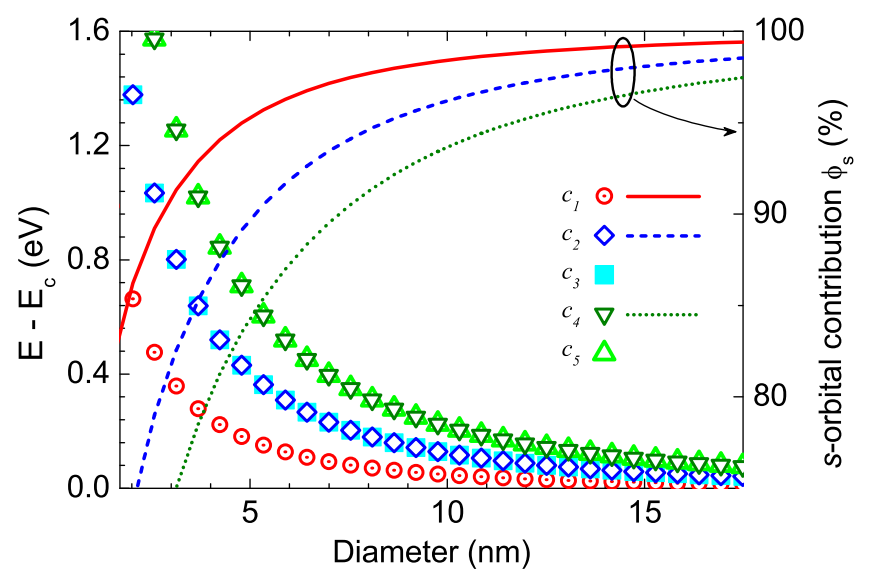

Figure 4. The size dependence of the lowest CB states of GaN NWs. The symbols are the energies relative to the bulk GaN CB edge. The lines represent the $s$-orbital contribution to the wave functions.

state in the hole picture) exhibits a change in its properties at that critical size. For NWs with diameter larger than $5 \mathrm{~nm}$, the highest-energy state has dominant $p_{\perp}$ character $\left(\phi_{\perp}\right.$ larger than $98 \%$ ), as the bulk band edge. However, when the diameter decreases below $5 \mathrm{~nm}$, the dominant character of the highest state becomes $p_{z}$. Moreover, this change in the atomic character is accompanied by a change in the orbital, envelope symmetry of the state, as is illustrated also in Fig. 5 by showing the cross-sectional graphs of the square of the wave functions for the highest-energy states of NWs with diameters $3.1 \mathrm{~nm}$ and $8.7 \mathrm{~nm}$. We recognize that the charge density of the state with dominant $p_{z}$-orbital contribution has only one maximum at the NW center, whereas the state with major contribution from $p_{\perp}$-orbitals, has a node in the center, thus exhibiting overall annular form. We thus conclude that the spatial confinement together with the mixed valence band structure create two different size ranges, for which the ground state hole state exhibits very different symmetry properties. This behavior of the hole ground state, which will have important consequences on the NW optical properties, (as will be shown below) is specific of GaN NWs and has its origins in the peculiar dispersion of the bulk band structure shown in Fig. 1. Various other crossings and anticrossings (see, e. g., the anticrossing around $8 \mathrm{~nm}$ between second (green) and third (pink) curves) of the size dispersion curves appear at lower energies, contributing to the complexity of the VB level structure.

The nontrivial impact of the size confinement effect on the one-dimensional band structure is expected to be apparent also in the subband dispersions. As confirmed by various calculations not shown, the conduction band structure is composed by successive subbands of similar curvature, that can be fitted to a parabolic expression for $\mathrm{k}$ smaller than $0.1 \AA^{-1}$. As commented before this behavior derives directly from the simplicity of the bulk band structure and can be accounted easily by simple EMA single-band approaches, with properly chosen parameters. In order to explore how the NW valence subband structure near the zone center is modified with the diameter, we present 


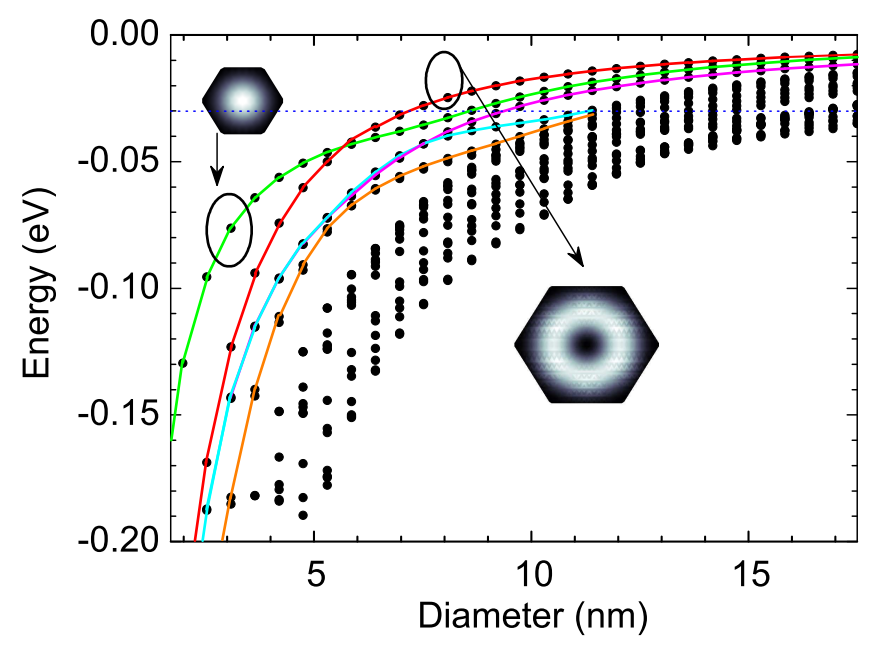

Figure 5. The size dependence of the 20 highest $k_{z}=0$ VB states of GaN NWs. The dots represent the energies relative to the bulk GaN VB edge, corresponding to $E_{1}$ in Fig. 1. The bulk band edge $E_{3}$ is also shown as a blue dotted horizontal line. The interpolating lines connecting the energy levels have been drawn by joining states with the same global symmetry. The square of the wave functions of the highest VB state is shown for two sizes: $d=3.1$ and $d=8.7 \mathrm{~nm}$. (Color online)

in Fig. 6 two illustrative examples corresponding to NW diameters $d=3.1$ and 8.1 $\mathrm{nm}$, lying on different sides of the crossing point, that delimitates different regions as discussed in Fig. 5.

Regarding the valence states, several kind of dispersion curves participate, even with a negative curvatures, and with the common characteristic of a negligible $s$ orbital contribution, less than $1 \%$. All these trends justify the necessity of multi-band approaches in the study of nitride NW's. In Fig. 6(a), we represent the bands of the NW with diameter $3.1 \mathrm{~nm}$. We can appreciate that the first valence band, with energy at $\Gamma$ point of $-77 \mathrm{meV}$, has a curvature associated with the band $E_{3}$ of GaN bulk (Fig. 1 (a)). On the contrary, the following state, with energy at $\Gamma$ point of $-120 \mathrm{meV}$, has now a flatter dispersion, in correspondence with the band $E_{1}$ of Fig. 1 (a). In the band structure represented in Fig. 6(b), of diameter $8.1 \mathrm{~nm}$, the band associated to $E_{3}$ is placed now at $-38 \mathrm{meV}$, and occupying the top of the valence band, there is a state with a dispersion similar to the band $E_{1}$, at the energy of $-25 \mathrm{meV}$. In this case, the confinement is reduced, bring together a considerable number of states in a shorter range of energy for both conduction and valence band.

In order to help in the understanding of the optical properties of NW, we have selected significant wave functions at the $\Gamma$ point, to represent them in Fig. 7 with a 2D mapping, and extract information about the optical transitions from the valence to conduction band. Thus, the first and second states of the conduction band correspond to $c_{1}$ and $c_{2}$, respectively, and the first valence states with predominant composition $p_{\perp}$-orbital and $p_{z}$-orbital are denoted with $v_{1, \perp}$ and $v_{1, z}$, respectively.

Concerning the effect of the electronic states on the optical response, the different 


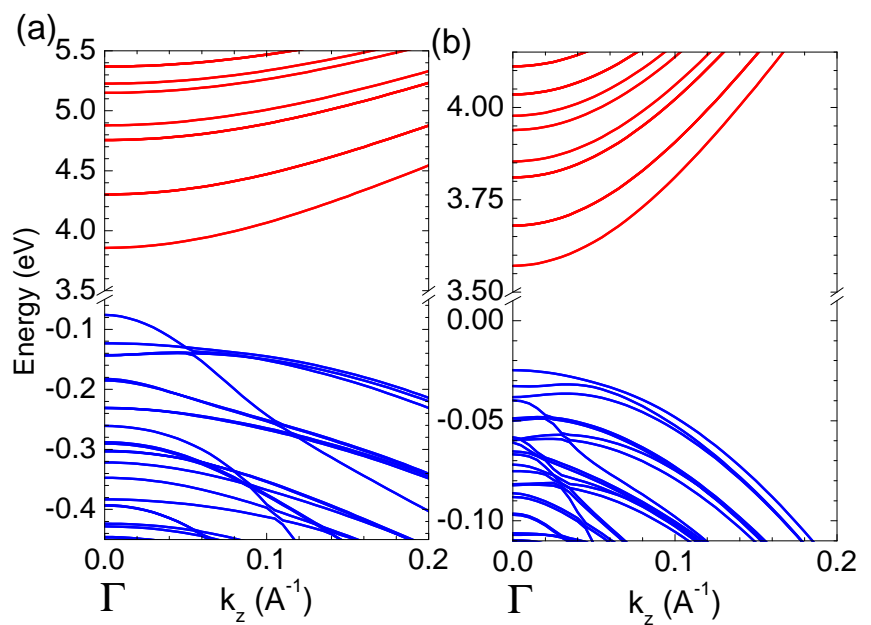

Figure 6. Band structure for NWs with diameter of (a) $3.1 \mathrm{~nm}$ and (b) $8.1 \mathrm{~nm}$.

envelope symmetry of the states $c_{1}$ and $v_{1, \perp}$ means a zero transition rate at the energy $3.585 \mathrm{eV}$, opposite to the transition from $v_{1, z}$ to $c_{1}$, at energy of $3.598 \mathrm{eV}$, where the states share symmetry. Commonly, these inefficient transitions as the $c_{1}-v_{1, \perp}$ are known as dark states or dark excitons. The existence of these states have already been predicted in $\mathrm{ZnO}$ NWs by density functional theory calculations[26]. In addition to the envelope symmetry, the orbital symmetry (expressed in the wave function character) plays a role due to the anisotropy of wurtzite NWs, as shown in our results of the electronic structure. Therefore, in the following study of the absorption, we have differentiated between light polarized in the $C$-plane $\left(\boldsymbol{e}_{\perp}\right.$-light) and light polarized along $C$-axis $\left(\boldsymbol{e}_{z}\right.$-light).

In Fig. 8 we present the absorption spectrum, calculated as described in Section 2, for the NW with size $3.1 \mathrm{~nm}$, associated to the band structure of Fig. 6(a). We have labeled the significant transitions in the energy range of interest to facilitate the interpretation. The characteristic one-dimensional density of states is visible in all the energy range for both polarizations. Concerning to $\boldsymbol{e}_{\perp}$-light, the absorption edge is shifted with respect to the NW band gap, located at $3.92 \mathrm{eV}$, due to the following reasons. First, the different envelope symmetry between states $v_{1, \perp}$ and $c_{1}$, and second, the $p_{z}$ character of the ground state of the valence band. The first important absorption peak is placed at $4.05 \mathrm{eV}$, being produced by the transition $v_{2, \perp}-c_{1}$, followed by the successive contributions of other excited states. Note that until $4.33 \mathrm{eV}$ all the transitions involved the ground conduction state. Concerning the $\boldsymbol{e}_{\boldsymbol{z}}$-light, the absorption edge and NW band gap coincides at $3.92 \mathrm{eV}$, as we can infer from the envelope symmetry of the states $v_{1, z}$ and $c_{1}$, both with the maximum of the wave function in the center of the NW, as shown in Fig. 5. The absorption peaks are also separated and well differentiated in comparison with the $\boldsymbol{e}_{\perp}$-light spectra.

In Fig. 9, the absorption of a NW of diameter $8.7 \mathrm{~nm}$ is presented. Now, the 1Ddensity of states is masked by the large amount of states although still remains visible $\boldsymbol{e}_{z}$-light. In the case of $\boldsymbol{e}_{\perp}$-light, the absorption edge is slightly blue-shifted relative to the 


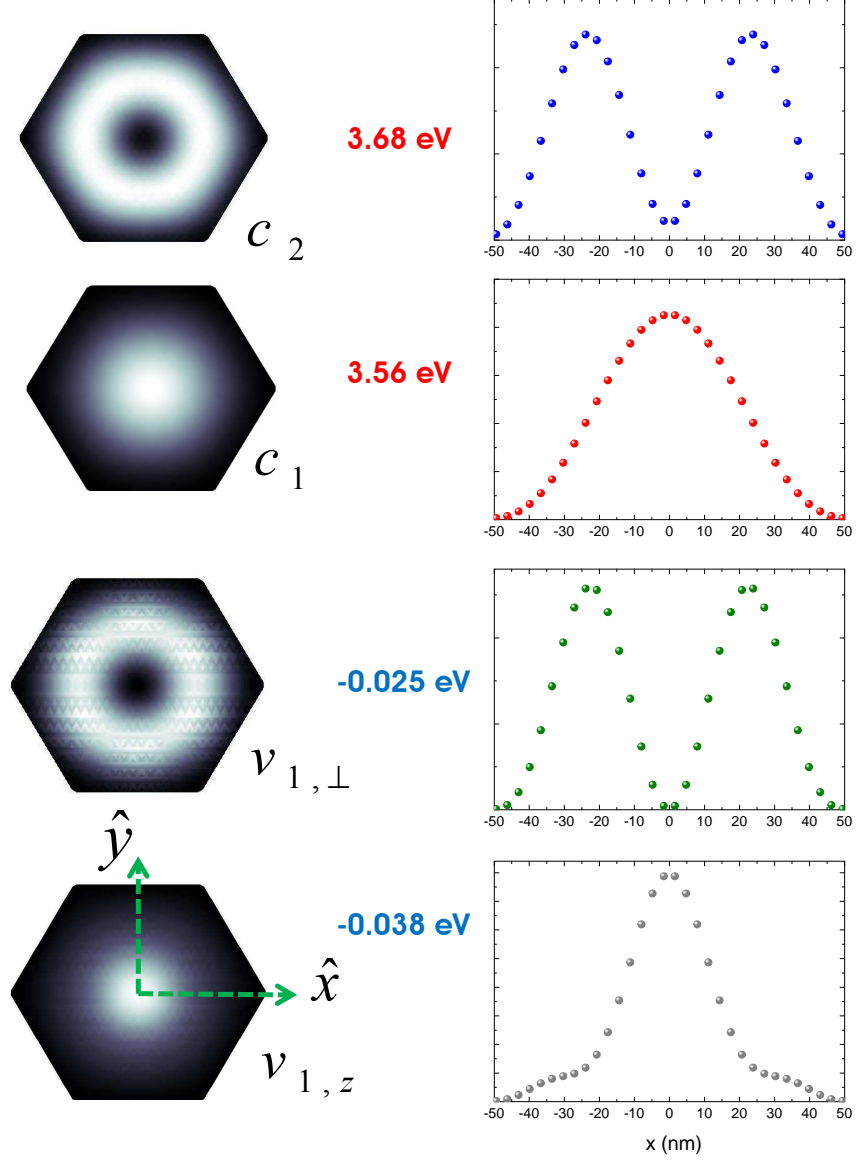

Figure 7. Charge density of several states of a GaN-NW with diameter $8.1 \mathrm{~nm}$. The states $e_{1}$ and $e_{2}$ belong to the conduction band and the states $h_{1 \perp}$ and $h_{1 z}$ belong to the valence band. The subindex indicates the state character. The right column shows the profile along $\hat{x}$-axis.

NW band gap due to the dark transition between both ground valence and conduction band states, but still takes place at lower energy (15 meV less) than $\boldsymbol{e}_{z}$-light, reversing the situation of Fig. 8. In the case of the $\boldsymbol{e}_{z}$-light spectra, the separation between peaks is still noticeable in comparison with the case of $\boldsymbol{e}_{\perp}$-light spectra. From both Figs. 8 and 9 is remarkable how with the unique effect of confinement, the polarization of the optical response can be altered. Similar polarizations cross-effect depending on the nanostructure size have been measured in very thin GaN/AlN quantum dots and GaN/AlN quantum wells, being the strain together with the confinement the responsible of the changes in the valence states order reported.[56, 57]

\subsection{AlN nanowires}

The previous study of the optical properties of GaN NW, where we have shown their link with the bulk band structure, can be used here to understand the optical properties of AlN NWs. 


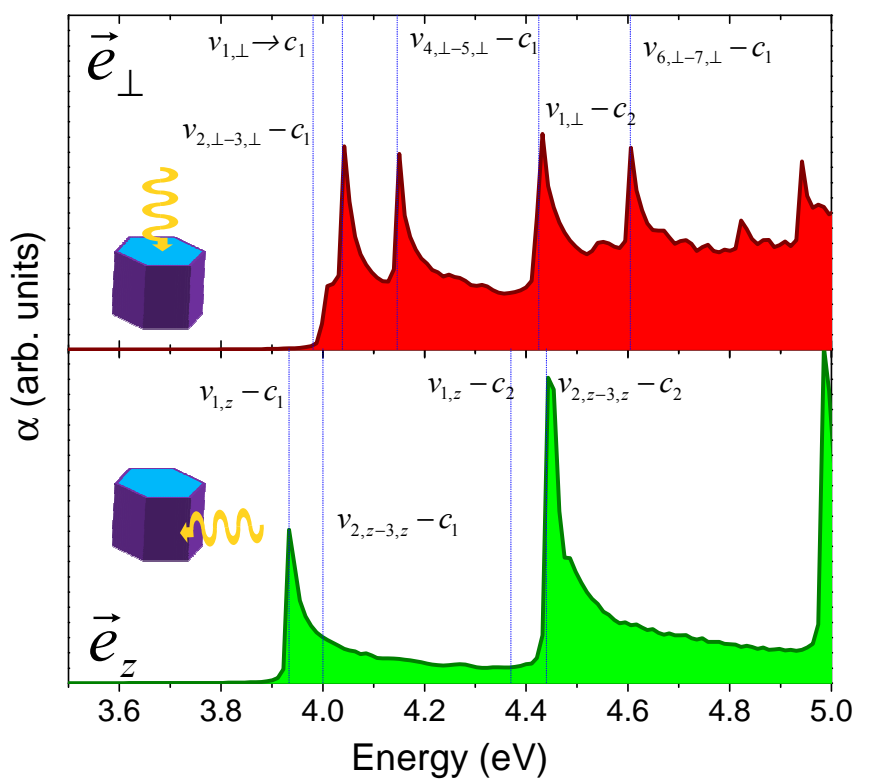

Figure 8. Optical absorption spectrum for GaN NWs of $3.1 \mathrm{~nm}$. Top: $\boldsymbol{e}_{\perp}$-light; bottom: $\boldsymbol{e}_{z}$-light. The dotted lines indicate relevant transitions. The left label is the predominant orbital for valence states and the right label number the conduction states.

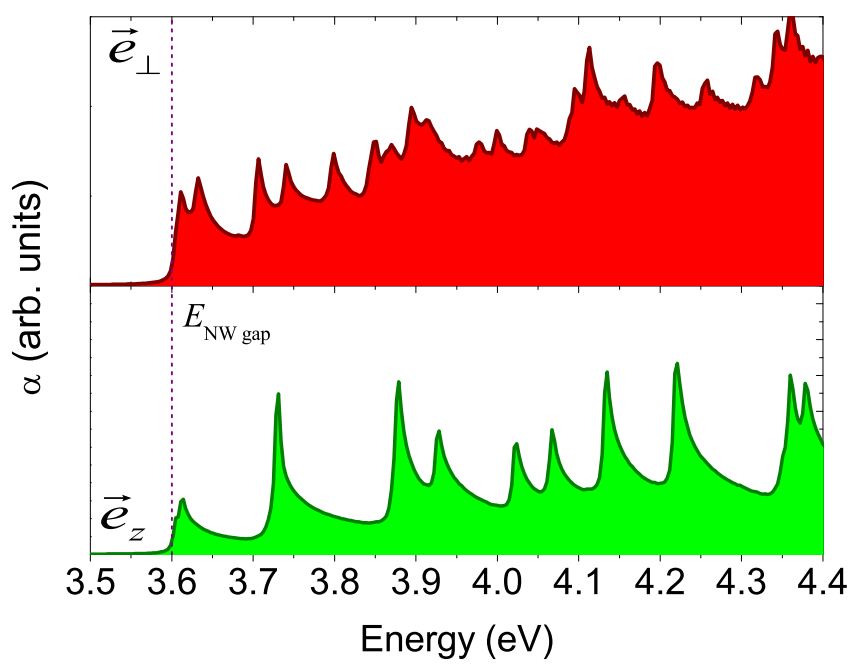

Figure 9. Idem as in Fig. 8 for a GaN NW of diameter $8.7 \mathrm{~nm}$.

We have seen in Section 2 that the AlN bulk valence band is substantially different than those of GaN. We recall that here the topmost VB state has a $p_{z}$-character $\left(\phi_{p_{z}}=100 \%\right)$. The splitting between the topmost VB state and the other VB states ranges roughly from 100 to $300 \mathrm{meV}$ (in our TB parameter is set in $\sim 120 \mathrm{meV}$ ). On the other hand, the spin-orbit splittings takes a small value, as seen in Fig. 1. Concerning the conduction band, the large band gap of $\mathrm{AlN}, \sim 6.2 \mathrm{eV}$, decouples almost totally the conduction and topmost valence band would have a behaviour similar to those of a 
single-band, as we will see.

Regarding the CB states behaviour when the NW size varies, we found that CB states energy at $k=0$ decreases in a similar way that in the case of GaN NWs. However, in this case, this behaviour is closer to the EMA prediction, and the energy of the CB state $c_{1}$, can be fitted to the following function:

$$
\epsilon_{c_{1}}=E_{c}+\frac{a}{d^{2}}-\frac{b}{d^{3}} \quad(\text { in } \mathrm{eV}),
$$

where $a=1.84 \pm 0.02, b=0.85 \pm 0.02$, and $E_{c}=6.2 \mathrm{eV}$. For small diameters, although the AlN band gap is very large, the strong quantum confinement deviates the energy dependence slightly from the EMA prediction for parabolic bands, $i$. e., from $1 / d^{2}$. We have corrected this overestimation by adding a cubic term, which it is only effective for diameters below $5 \mathrm{~nm}$. For larger NWs, the expression given by EMA works reasonably well. On the other hand, the $s$-orbital contribution to the state $c_{1}$ has found to be $\phi_{s}>95 \%$ for all the cases, which confirms a single-band character for the CB state $c_{1}$ of AlN NWs.

On the other hand, the analysis of the VB states is more interesting because their impact on the optical properties. Figure 10 shows the evolution of the VB states with the NW diameter. Due to the large splitting among the VB states with $p_{z}$-character and $p_{\perp}$-character in the bulk band structure (see Fig. 1), we can now label the states as $v_{z, n}$ ( $n$ in increasing order of energy) until the first VB state with $p_{\perp}$-character. In Fig. 10 the VB states $v_{z, 1}, v_{z, 2}$ and $v_{z, 3}$ have been represented with black circles and the two VB states $v_{\perp, 1}$ and $v_{\perp, 2}$ with red circles. With the aim of having a clear picture of the evolution, we have eliminated all the states between these two sets of energies. Contrary to the case of GaN, we can observe the absence of the complex crossings and anti-crossings observed in the valence band of GaN NWs (see for instance Fig. 5). Now, we can fit the energy of the VB state $v_{z, 1}$ by the same function of Eq. (9), obtaining the following results:

$$
\epsilon_{v_{1}}=-\frac{417}{d^{2}}+\frac{159}{d^{3}} \quad(\text { in } \mathrm{meV})
$$

where the coefficients error is $3 \mathrm{meV}$. The fitting is represented in Fig. 10 with a solid cyan line. Other differences with previous results come from the symmetry of the wave functions. In Fig. 10, we have plotted the charge density of the VB states $v_{z, 1}$ and $v_{\perp, 1}$. Here, the state $v_{z, 1}$ has the density concentrated at the NW core, decaying smoothly until be zero at the border. This shape of the charge density amply matches with that of the first $\mathrm{CB}$ state (not shown here). This agrees with the expected EMA result for the first confined state in a NW when the Hamiltonian is solved in the single-band approximation. In addition, the state $v_{\perp, 1}$ of $p_{\perp}$-character has the annular profile also exhibited by the topmost VB state of GaN NWs, due to the mixing between contiguous states, as explained before.

After the analysis of the electronic structure of AlN NWs, we do not expect substantial changes in the optical absorption when the NWs size varies. For this reason, 


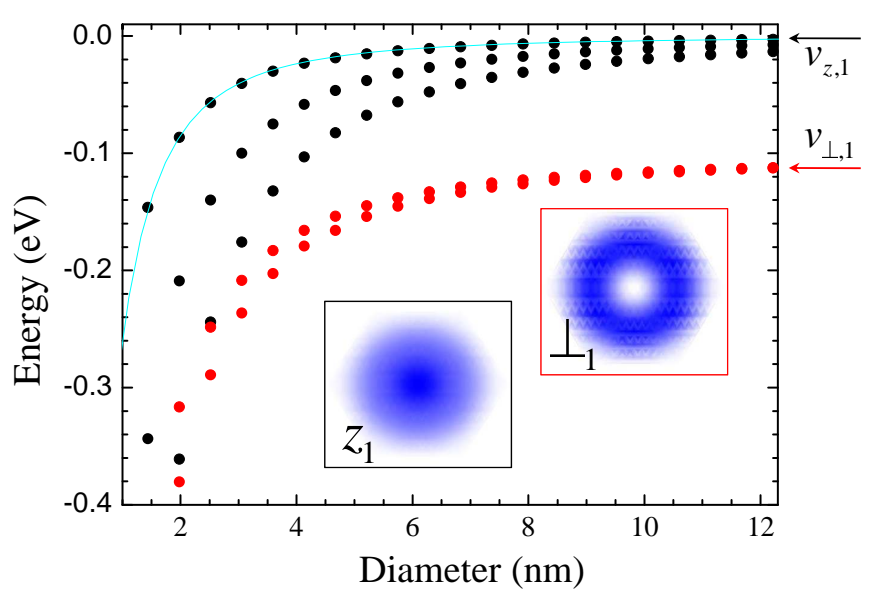

Figure 10. Valence band states as a function of the AlN nanowire diameter. Black dots are the first three valence states with character $p_{z}>95$. The red dots are the first two valence states with character $p_{\perp}>95$. The wave functions of the first state of each class are given for a diameter of $8 \mathrm{~nm}$. The solid line is a fitting with the function given in the main text.

in Fig. 11 we have represented the absorption of a NW of diameter $5.3 \mathrm{~nm}$, for the light polarizations in-plane and on-axis. The one-dimensional density of states is indubitably recognized, observing a larger number of transitions in the case of the $\boldsymbol{e}_{\perp}$-light, as usual. The difference with the other spectra (see Figs. 8 and 9) arises in the relative position of the absorption edges obtained for different light polarizations. Hence, the absorption edge for $\boldsymbol{e}_{z}$-light is clearly located at an energy much lower than its counterpart in the case of $\boldsymbol{e}_{\perp}$-light, being separated by $143 \mathrm{meV}$ for the NW size shown here. Still the second relevant transition for on-axis light takes places at lower energy than the absorption edge of the in-plane polarization $(129 \mathrm{meV}$ blue-shifted with respect to the first transition). Considering the evolution of the VB states shown in Fig. 10, we can argue that the optical spectra for thicker AlN NWs will be very similar to the one displayed here, with the only difference of a ripple profile. Also, the shift between absorption edge of the different polarizations will tend to the bulk AlN $\Delta_{c f}$, as the NW size increases.

\section{Conclusions}

We have presented an atomistic and empirical model of the band structure of GaN and AlN nanowires, including the crystal-field splitting and the spin-orbit interaction. Then, we have explored the evolution of the nanowire states with size, the charge density and the optical response. We have studied how the confinement influences in GaN and AlN nanowires until $20 \mathrm{~nm}$. In the case of GaN NWs, the absorption edge changes the polarization around $7 \mathrm{~nm}$, in concordance with the ab initio results of Schrier.[26] The differences in the optical properties between GaN and AlN nanowires are explained in function of the wave function character, and the optical response spectra confirms 


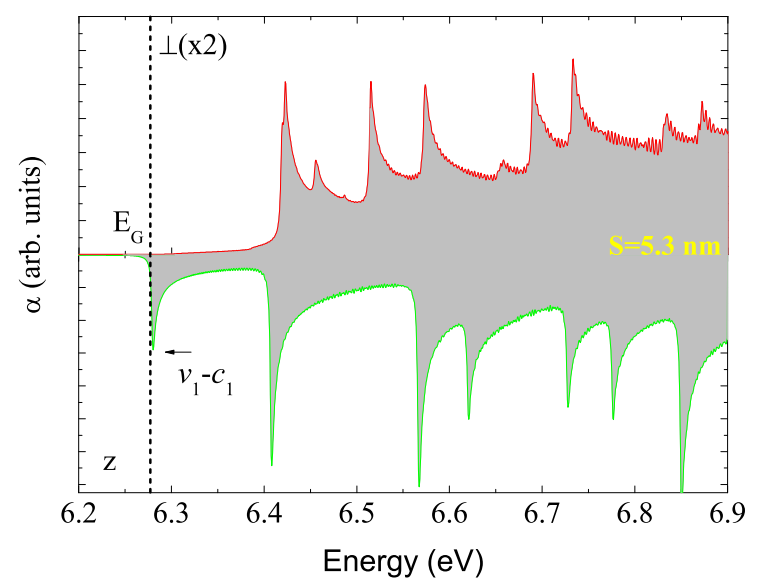

Figure 11. Absorption spectra of a AlN NW with diameter $5.3 \mathrm{~nm}$, for the light polarizations in-plane $\left(\boldsymbol{e}_{\perp}\right)$ and on-axis $\left(\boldsymbol{e}_{z}\right)$.

favorable absorption for $\boldsymbol{e}_{z}$-light for AlN nanowires.

\section{Acknowledgments}

This work was supported the ERANET project "NanoSci-ERA: NanoScience in the Europen Research Area" and ACDET II of the EU FP6.

[1] Y. Huang, X. Duan, Y. Cui, L. J. Lauhon, K. H. Kim, and C. M. Lieber, Science 294, 1313 (2001).

[2] S. Kako, C. Santori, K. Hoshino, S. Gótzinger, Y. Yamamoto, and Y. Arakawa, Nature Materials 5, 887 (2006).

[3] O. Landre, C. Bougerol, H. Revevier, and B. Daudin, Nanotechnology, 20, 415602 (2009).

[4] C. Denker, J. Malindretos, F. Werner, F. Limbach, H. Schuhmann, T. Niermann, M. Seibt, and A. Rizzi. Phys. Stat. Sol. (c) 5, 1706 (2008).

[5] G. R. Yazdi, P. O. A. Persson, D. Gogova, R. Fornari, L. Hultman, M. Syvajarvi, and R. Yakimova, Nanotechnology 20, 495304 (2009).

[6] H. W. Kim, M. A. Kebede, and H. S. Kim, Appl. Surf. Science 2557221 (2009).

[7] O. Landre, V. Fellmann, P. Jaffrennou, C. Bougerol, H. Renevier, A. Cros, and B. Daudin, Appl. Phys. Lett. 96, 061912 (2010).

[8] A. I. Hochbaum, R. Chen, R. D. Delgado, W. Liang, E. C. Garnett, M. Najarian, A. Majumdar, and P. Yang. Nature 451, 163 (2008).

[9] R. Calarco, M. Marso, T. Richter, A. I. Aykanat, R. Meijers, A. Hart, T. Stoica, and H. Lúth, Nano Lett. 5, 981 (2005).

[10] J. B. Schlager, N. A. Sanford, K. A. Bertness, J. M. Barker, A. Roshko, and P. Blanchard. Appl. Phys. Lett. 88, 213106 (2006).

[11] Q. Zhao, H. Zhang, X. Xu, Z. Wang, J. Xu, and D. Yu, Appl. Phys. Lett. 86, 193101 (2005).

[12] O. Jani, I. Ferguson, C. Honsberg, and S. Kurtz. Appl. Phys. Lett. 91, 132117 (2007).

[13] Y. B. Tang, Z. H. Chen, H. S. Song, C. S. Lee, H. T. Cong, H. M. Cheng, W. J. Zhang, I. Bello, and S. T. Lee, Nanolett. 84191 (2008).

[14] Y. Huang, X. Duan, Y. Cui, and C. M. Lieber, Nanolett. 2, 101 (2002).

[15] T. Kuykendall, P. Ulrich, S. Aloni, and P. Yang, Nature Mater. 6, 951 (2007). 
[16] M. S. Gudiksen, J. Wang, and C. M. Lieber. Chemistry B 106, 4036 (2002).

[17] A. H. Chin, T. S. Ahn, H. L. Li, S. Vaddiraju, C. J. Bardeen, C. Z. Ning, and M. K. Sunkara. Nanolett. 7, 626 (2007).

[18] O. Stier, M. Grundmann, and D. Bimberg, Phys. Rev. B 59, 5688 (1999).

[19] L. W. Wang and A. Zunger, Phys. Rev. B 59, 5678 (1999).

[20] X. Z. Li and J. B. Xia, Phys. Rev. B 66, 115316 (2002).

[21] A. V. Maslov and C. Z. Ning, Phys. Rev. B 72, 125319 (2005).

[22] A. Zunger, Phys. Stat. sol. (a) 190, 467 (2002).

[23] G. Bester and A. Zunger, Phys. Rev. B 71, 045318 (2005).

[24] T. Akiyama, A. J. Freeman, K. Nakamura, and T. Ito, Journal of Physics: Conference Series 100 052056 (2008).

[25] Y. Wu, G. Chen, H. Ye, Y. Zhu, and S. H. Wei, J. Appl. Phys. 104, 084313 (2008).

[26] J. Schrier, D. O. Demchenko, and L. W. Wang, Nanolett. 7, 2377 (2007).

[27] S. Schulz, S. Schumacher, and G. Czycholl, Phys. Rev. B 73, 245327 (2006).

[28] Y. M. Niquet, Phys. Rev. B 74, 155304 (2006).

[29] M. P. Persson and A. Di Carlo, J. Appl. Phys. 104, 073718 (2008).

[30] A. Molina-Sánchez, A. García-Cristóbal, A. Cantarero, A. Terentjevs, G. Cicero, Phys. Rev. B 82 165324 (2010).

[31] J. C. Slater and G. F. Koster, Phys. Rev. 94, 1498 (1954).

[32] A. Kobayashi, O. F. Sankey, S. M. Volz, and D. Dow, Phys. Rev. B 28, 935 (1983).

[33] P. O. Löwdin, J. Chem. Phys. 18, 365 (1950).

[34] O. Marquardt, D. Mourad, S. Schulz, T. Hickel, G. Czycholl, and J. Neugebauer, Phys. Rev. B 78, 235302 (2008).

[35] T. Yang, S. Nakajima, and S. Sakai, Jpn. J. Appl. Phys. 4, 5912 (1995).

[36] J. M. Jancu, F. Bassani, F. Della Sala, and R. Scholz, Appl. Phys. Lett. 81, 4838 (2002).

[37] P. Y. Yu and M. Cardona, Fundamentals of Semiconductors (Springer, Berlin, 1974)

[38] R. Enderlein and N. J. Horing, Fundamentals of Semiconductor Physics and Devices (World Scientific, Singapore and River Edge, 1997)

[39] J. Schrier, K. B. Whaley, Phys. Rev. B 67, 235301 (2003).

[40] S. Schulz, S. Schumacher, and G. Czycholl, European Phys. J. B 64, 51 (2008).

[41] D. J. Chadi, Phys. Rev. B 16, 790 (1977).

[42] L. C. Lew Yan Voon, M. Villatzeu, M. Cardona, and N. E. Christensen. Phys. Rev. B 53, 10703 (1996).

[43] D. W. Jenkins and J. D. Dow, Phys. Rev. B 39, 3317 (1989).

[44] M. Graf and P. Vogl, Phys. Rev. B 51, 4940 (1995).

[45] P. Rinke, M. Winkelnkemper, A. Qteish, D. Bimberg, J. Neugebauer, and M. Scheffler. Phys. Rev. B 77, 075202 (2008).

[46] Y. Taniyasu, M. Kasu, and T. Makimoto, Appl. Phys. Lett. 90, 261911 (2007).

[47] S. K. Pugh, D. J. Dugdale, S. Brand, and R. A. Abram, Semicond. Sci. Technol. 14, 23 (1999).

[48] I. Vurgaftman, J. R. Meyer, and L. R. Ram-Mohan, Appl. Phys. Rev. 89, 5815 (2001).

[49] R. Songmuang, O. Landré, and B. Daudin, Appl. Phys. Lett. 91, 251902 (2007).

[50] K. A. Bertness, A. Roshko, L. M. Mansfield, T. E. Harvey, and N. A. Sanford, J. Cryst. Growth 300, 94 (2007).

[51] T. Kuykendall, P. J. Pauzauskie, Y. Zhang, J. Goldberger, D. Sirbuly, J. Denlinger, and P. Yang. Nature Materials 3, 524 (2004).

[52] J. E. Northrup and J. Neugebauer, Phys. Rev. B 53, R10477 (1996).

[53] M. Cruz, M. R. Beltrán, C. Wang, J. Tagüeña-Martínez, and Y. G. Rubo, Phys. Rev. B 59, 15831 (1999).

[54] W. A. Harrison, Electronic Structure and the Properties of Solids (Dover, New York, 1989).

[55] N. Drakos, ARPACK Users Guide: Solution of Large Scale Eigenvalue Problems with Implicitly Restarted Arnoldi Methods. Computer Based Learning Unit, University of Leeds. 
[56] A. Molina, A. García-Cristóbal, and A. Cantarero. Microelectronics J. 40, 418 (2009).

[57] R. Mata, A. Cros, J. Budagosky, A. Molina-Sánchez, N. Garro, A. García-Cristóbal, J. Renard, S. Founta, B. Gayral, E. Bellet-Almaric, C. Bougerol, and B. Daudin. Phys. Rev. B 82, 125405 (2010). 